\title{
ESTUDIO SOBRE INCIDENCIA Y CAUSAS ECONOMICO-SOCIALES DE LA DISTROFIA EN UN CENTRO INFANTIL
}

\author{
Dr. ARTURO GAILLO y Srta. ADELINA BELLO \\ Cátedra de Pediatria def Prof. Adalberto Steeger, Hospital "San Juan de Dios" \\ y Cátedra de Higiene Materna e Infantif dei Prof. Guillermo Adriasola, \\ Escuela de Salubridad, Santiago.
}

El problema de las distrofias sigue siendo una de las causas más importantes de mortalidad y morbilidad en nuestro pais. Sorprende por eso que persistan todavía una serie de hechos importantes que son desconocidos. Uno de ellos es la magnitud del problema en un plano nacional, ya que casi toda la información que exista al respecto proviene de medios hospitalarios ${ }^{1.2 .3}$, que representan una muestra muy seleccionada. Por otra parte, en las estadísticas oficiales del Sub Depto. de Bio-Estadística del S. N. S., las distrofias se incluyen en el rubro 722 , de "Inadaptación al régimen alimenticio", que representa alrededior de un $10 \%$ del total de causas de muerte en el menor de 1 año 4. Estas cifras tienen, sin embargo, escaso valor, ya que el diagnóstico de distrofia no se anota generalmente en los certificados de defunción de lactantes fallecidos por diarreas infantiles, enfermedades respiratorias o infecciosas, etc., en que la deficiencia del estado nutritivo actuó en forma muy importante.

Otro aspecto que ha sido objeto de numerosas discusiones entre los pediatras del país ${ }^{5}$ ha sido el de la posibilidad de acción de los servicios médicos sobre los factores económico-sociales presentes en los hogares de los niños distróficos, sin haber llegado todavía a conclusiones definitivas.

El objeto de este trabajo ha sido precisamente presentar el resultado de algunas observaciones sobre incidencia y factores económico-sociales efectuadas en un grupo de lactantes asistentes a un Centro Infantil, en que se ensayó una nueva modalidad de atención ${ }^{\circ}$, y en que los Iactantes distróficos estuvieron sometidos a un tratamiento especial.

Material y método de trabajo. El material estuvo constituído por los lactantes nacidos en el Sector $N^{9} 10$ (correspondiente a la Población Simón Bolívar) de Ia Comuna de Quinta Normal en los meses de Marzo a Agosto inclusives de 1957, e inscritos en el ex-Centro de Salud $\mathrm{N}^{0} 5$ de la Zonal de Santiago. De ellos, pudo llevarse un buen control hasta el año de edad de 158 lactantes.

La supervisión de salud se efectuó periódicamente por médico y enfermera, en la forma descrita en la publicación aludida, especialmente en cuanto a su progreso ponderal. Para tener una visión más gráfica de este proceso se agregó a cada ficha la hoja de control de desarrollo para el niño chileno, confeccionada por el Dr. Farncisco Mardones. De esta hoja se aprovechó sólo la curva de peso, que fué dibujada por la auxiliar de enfermería, y cuyo análisis permitió estudiar la distribución de las diferentes modalidades de progreso ponderal en el ler. año de vida, que constituye la $1^{\text {a }}$ parte del trabajo.

A todos aquellos lactantes que el pediatra calificaba como distróficos, así como a aquellos en que la gráfica ponderal demostraba descenso o estacionamiento del peso durante 2 controles o más, se les incluía en el "plan de distróficos". Este consistía fundamentalmente en una acción intensiva a domicilio y discusión de cada caso por el equipo formado por el pediatra, enfermera, asistente social y auxiliar. La enfermera pudo dedicar mayor número de visitas a estos niños, por cuanto suprimió totalmente las visitas domiciliarias de rutina a niño sano. Los distróficos fueron así visitados cada 10 a 15 días como término medio. En cada hogar se estudiaban las factores económicos, culturales, previsionales, asistenciales 0 de otro orden que pudieran influir en la distrofia, se intentaba su solución y se efectuaba una labor educativa intensiva, sobre temas como alimentación, higiene, inmunizaciones $\mathrm{y}$ otros. $\mathrm{E} 1$ número de distróficos que pudieron ser manejados en esta forma alcanzó a 27. El propósito de efectuar regulammente verdaderas reuniones clinicas del equipo para analizar cada caso, se vió frustrado por la ausencia de una asistente social, y la gran congestión 
TABLA NO 1

DISTRIBUCION DE 158 LACTANTES SEGUN FE TIPO DE CURVA PONDKRAL

Ex-Centro de Salud No 5 - 2257-1956.

\begin{tabular}{|c|c|c|c|c|c|c|c|c|c|c|}
\hline & & \multirow[b]{2}{*}{ No } & \multirow[b]{2}{*}{$\%$} & & \multirow{2}{*}{$\frac{\text { Distrotia }}{\text { No }}$} & \multicolumn{2}{|c|}{ infantil a $80 \%$} & \multicolumn{3}{|c|}{ Distrofla dafantil a 70\% } \\
\hline & & & & 1 & & | & $\%$ & No & I & $\%$ \\
\hline $\begin{array}{l}\text { Normal } \\
\text { Id., a ntvel bajo } \\
\text { Descenso } \\
\text { Recup. parclal } \\
\text { Recup. total } \\
\text { Elimingdos }\end{array}$ & $\begin{array}{l}\text { Tlpo } 1 \\
\text { Tipo } \\
\text { Typo } \\
\text { Tlpo } \\
\text { Tlpo } \\
\text { Thpo }\end{array}$ & $\begin{array}{r}66 \\
26 \\
38 \\
28 \\
4 \\
2\end{array}$ & $\begin{array}{r}41.7 \\
18.5 \\
34,0 \\
13,9 \\
2,5 \\
1,3\end{array}$ & $\mid$ & $\begin{array}{l}\overline{-} \\
\overline{12} \\
\frac{11}{2}\end{array}$ & 1 & $\begin{array}{l}\overline{7,6} \\
6, \theta \\
\overline{6,8}\end{array}$ & $\overline{\overline{5}}$ & 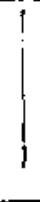 & $\begin{array}{l}\overline{7} \\
3,2 \\
4,4 \\
1,3\end{array}$ \\
\hline TOTAL & & 158 & 100,0 & i & 23 & li & 14,5 & 14 & $i$ & 8,9 \\
\hline
\end{tabular}

de público ( 30 a 40 consultas diarias para 3 horas pediatra). Sólo fué posible mantener un intercambio informal de opiniones entre médico y enfermera, a veces también con la auxiliar, a propósito de la asistencia a consulta médica de cada uno de estos niños. EI resultado del análisis de estos factores económico-sociales y de la labor desarrollada en relación con ellos es la materia de la $2^{a}$ parte del trabajo.

EI análisis de esta Tabla es causa de seria preocupación, ya que muestra que el $40,4 \%$ de nuestros lactantes presentan un descenso de peso de cierta consideración en el primer año de vida. Es, sin embargo, alentador que el $16,4 \%$ presenta luego una recuperación ponderal en el mismo período y que en el $2,5 \%$ esta recuperación sea total.

El descenso ponderal fué en algunos casos sólo discreto, en forma que casi no puede catalogarse a estos niños como distróficos. De ellos hemos separado los que presentaron un descenso ponderal que los colocó en niveles inferiores al $80 \%$. ElIos fueron 37 , o un $23,4 \%$ de los cuales 20 (54\%) se recuperaron. Los descensos de peso más acentuado con descensos bajo el $70 \%$, fueron sólo 14 , o un $8,9 \%$, de los cuales se recuperaron $9(64 \%)$. Vemos así que los distróficos más avanzados se recuperaron en mayor proporción.

Frecuencia de la distrofia. Más que en términos de distrofia, abordamos el problema desde el punto de vista del crecimiento del lactante, medido por su evolución ponderal, en relación con las curvas standard fabricadas por Mardones. Pese a que el proceso de crecimiento es esencialmente individual y presenta características propias para cada lactante, pudimos clasificar nuestros niños en 4 tipos de evolución ponderal, que son los sigujentes:

1. Crecimiento normal, es decir, niños cuyo peso se mantuvo siempre en niveles superiores al $89 \%$. En este grupo se distinguen aquellos que siguieron estrictamente su línea de evolución, de aquellos que presentaron descensos discretos en el curso del año, pero sin bajar su peso del $90 \%$ de lo normal. El primer subgrupo comprendió a 46 lactantes y el $2^{\circ}$ a 20 , con un total de 66 , ejemplos de los cuales se presentan en el gráfico 1.

2 . Crecimiento normal, pero a bajo nivel, en que se trata de lactantes con pesos bajos desde el ingreso o desde el nacimiento (una buena proporción de ellos por prematurez) y que mantienen su peso en la misma linea de crecimiento $d \mathfrak{u}$ rante todo el primer año de vida. Este grupo sumó a 26 lactantes, de los cuales se presentan los ejemplos más típicos en el gráfico 2.

3. Descenso de la curva ponderal sin recuperación posterior, a niveles inferiores al $90 \%$ de la normalidad, que constituyen el grupo más claro de distróficos. Sumaron un total de 38 , cuyos ejemplos más típicos se presentan en el gráfico 3 . En varios de ellos el descenso ponderal fue provocado por algún cuadro mórbido, como sarampión, dispepsia, tuberculosis pulmonar, y 3 tuvieron que ser hospitalizados. Cuatro de ellos ingresaron a la observación con pesos ya bajos, descenso que se acentuó posteriormente.

4. Descenso de la curva ponderal a un nivel inferior al $90 \%$, con recuperación parcial o total posterior. La mayoría de ellos mostraron recuperación sólo parcial 


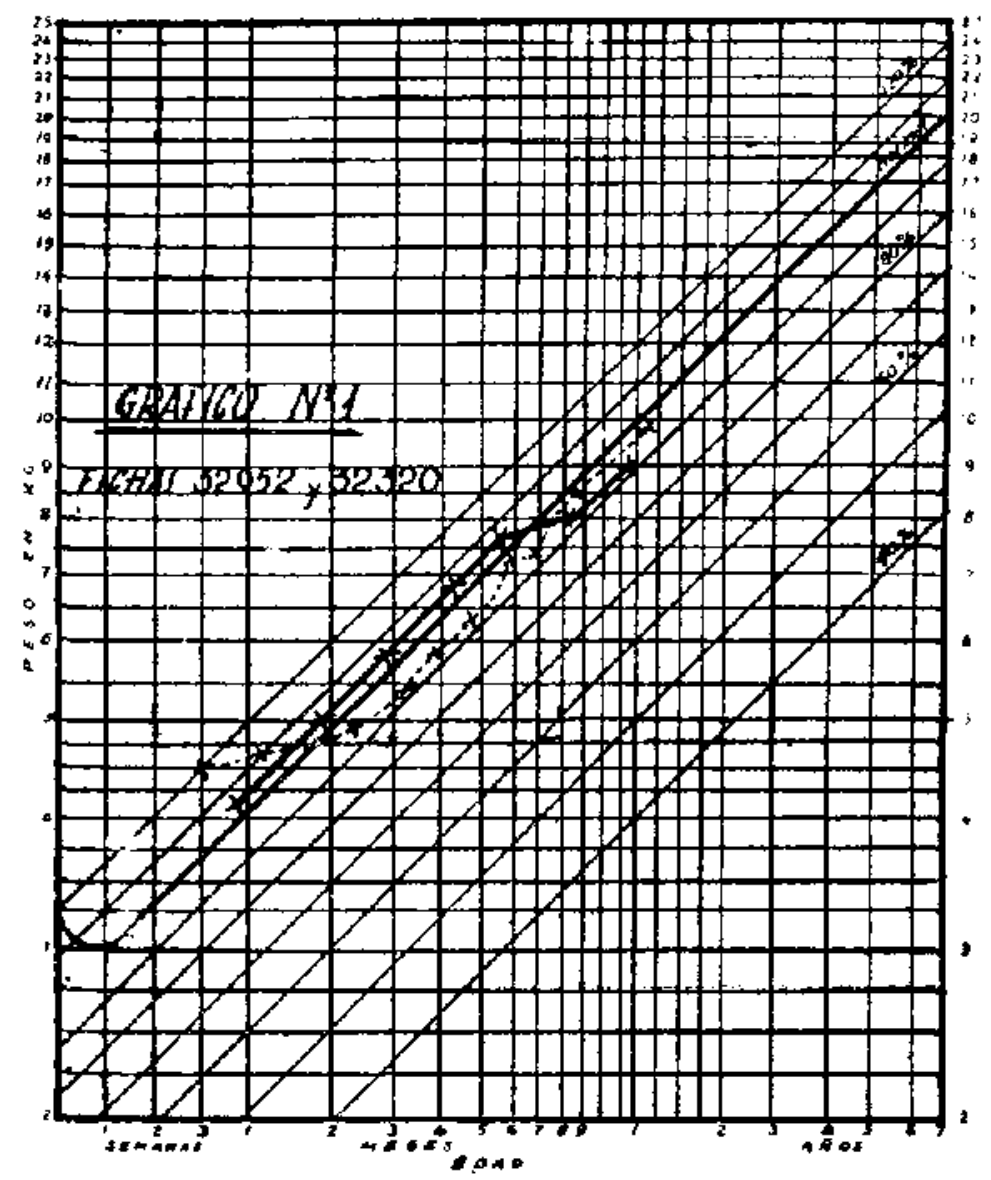

con un número total de 22 , cuyos ejemplos se presentan en el gráfico 4 . Tres de ellos tenían pesos bajos desde el ingreso, $y$ en 4 casos los controles hechos en el $2^{\circ}$ año de vida mostraron eutrofia. La recuperación total, después de un período variable de pesos subnormales, se observó en 4 casos, de los cuales uno era distrófico desde su ingreso. Los ejemplos de este subgrupo se aprecian en el gráfico 5.

El resumen de la distribución de los casos observados en los 4 grupos se pre. senta en la tabla 1. Debemos advertir que 2 lactantes fueron eliminados por haber sido supervisados por un tiempo muy corto.

Análisis de factores económico-sociales. Hemos dicho que el criterio que usamos para incluir a un lactante en el plan especial para distróficos fué bastante amplio ya que bastó para ello que se produjera un descenso de peso mantenido por 2 o más consultas, y sin esperar la comprobación de disergia u otros signos y sin- tomas de distrofia. No nos detendremos aqui en el análisis de las causas inmediatas de distrofia (alimentación, morbilidad, etc.) ni el tratamiento médico de elias, ya que estos temas son bien conocidos por todos los pediatras, y han sido objeto de numerosas presentaciones en esta Sociedad.

Nuestra investigación tiene por objeto mostrar 2 aspectos:

a) El análisis de los factores ambientales y económico-sociales que se encontraban presente en cada caso, actuando como causas profundas de la distrofia. Pensamos que dentro de la complejidad y de la íntima interrelación de estos factores, es posible, en una proporción de casos, individualizar una causa principal, sobre la que a veces es posible actuar, restituyendo asi la situación de ese hogar a la normalidad. Por el gran volumen de trabajo que pesaba sobre la enfermera y la ausencia de una asistente social en el equipo, este análisis sólo pudo ser efec- 


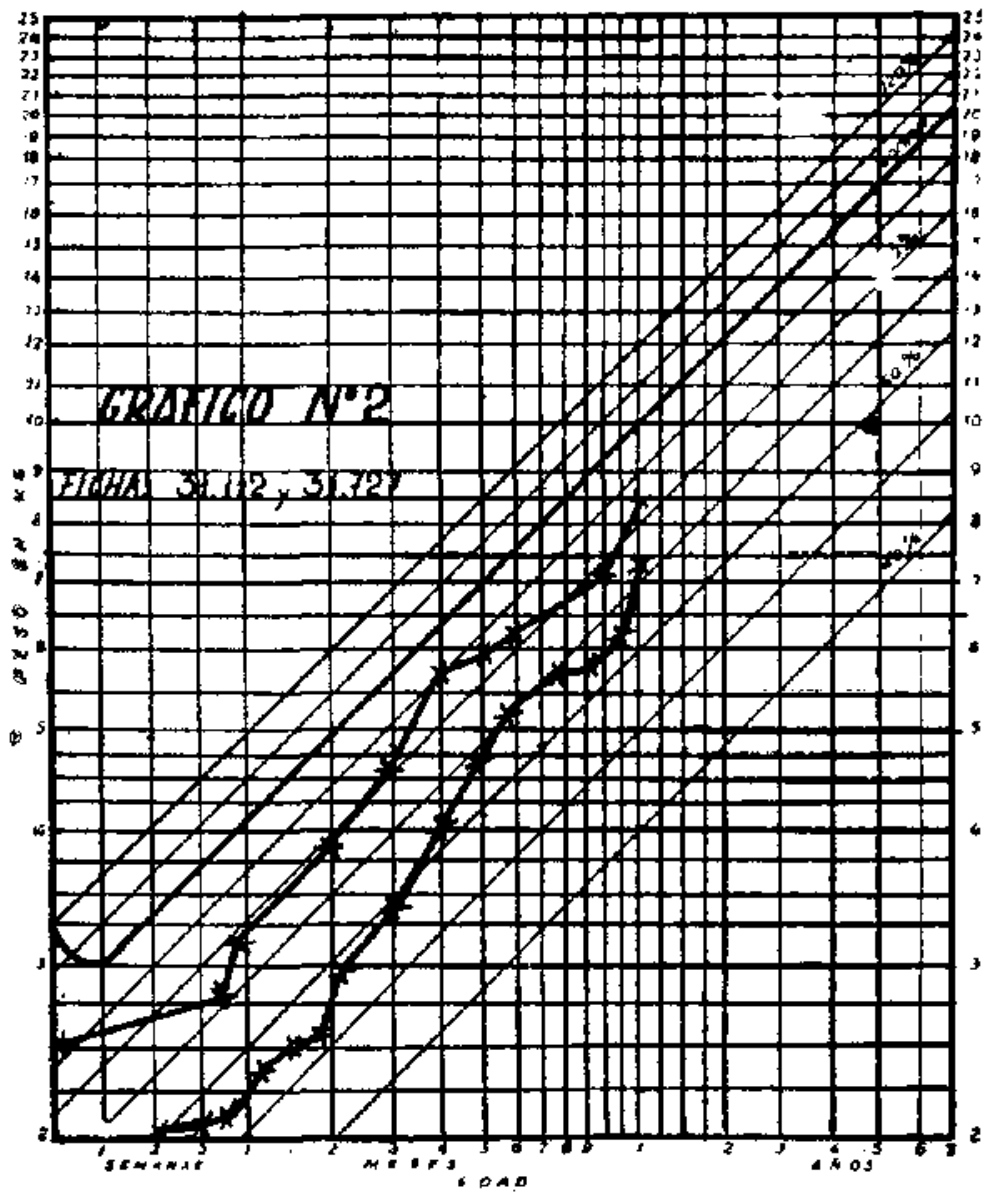

tuado con superficialidad, y se esquematizó en los siguientes puntos:

Situación económica de la familia.

Personalidad de la madre y/o otros componentes del grupo familiar, incluyendo nivel educacional, madurez intelectual y emocional, sentido de responsabiIidad.

Situación previsional y asistencial, en relación especialmente con la recepción de leche.

Condiciones de Higiene Ambiental y otras causas.

b) Establecido to que podríamos 1lamar el diagnóstico económico-social de la distrofia en algunos casos, se trató de soIucionar el problema principal y los agregados, en la medida de nuestras posibilidades, manteniendo siempre como objetivo final, la recuperación de la distrofia del lactante.
Los resultados del análisis son los siguientes :

1. La situación económica de la familia fué deficiente en 19 de los 27 casos estudjados, calificándose de pésima en 7 . Como caso máximo tuvimos el de una familia que durante varios días se alimentó sólo de té y de una sopa fabricada con pan y las cáscaras de las papas que botaban en el vecindario. Dentro de este grupo, sobresalieron dos tipos principales de situaciones. El primero fué aquel en que el jefe del hogar se encontraba cesante o presentaba otros problemas de trabajo, que comprendió a 8 familias. Caso típico fué el de un inspector de Liceos trasladado a Antofagasta, con su decreto en trámite y sin pago durante varios meses. El segundo correspondió a hijos de madres solteras sin protección económica, que vivían casi todas allegadas a otras familias. 


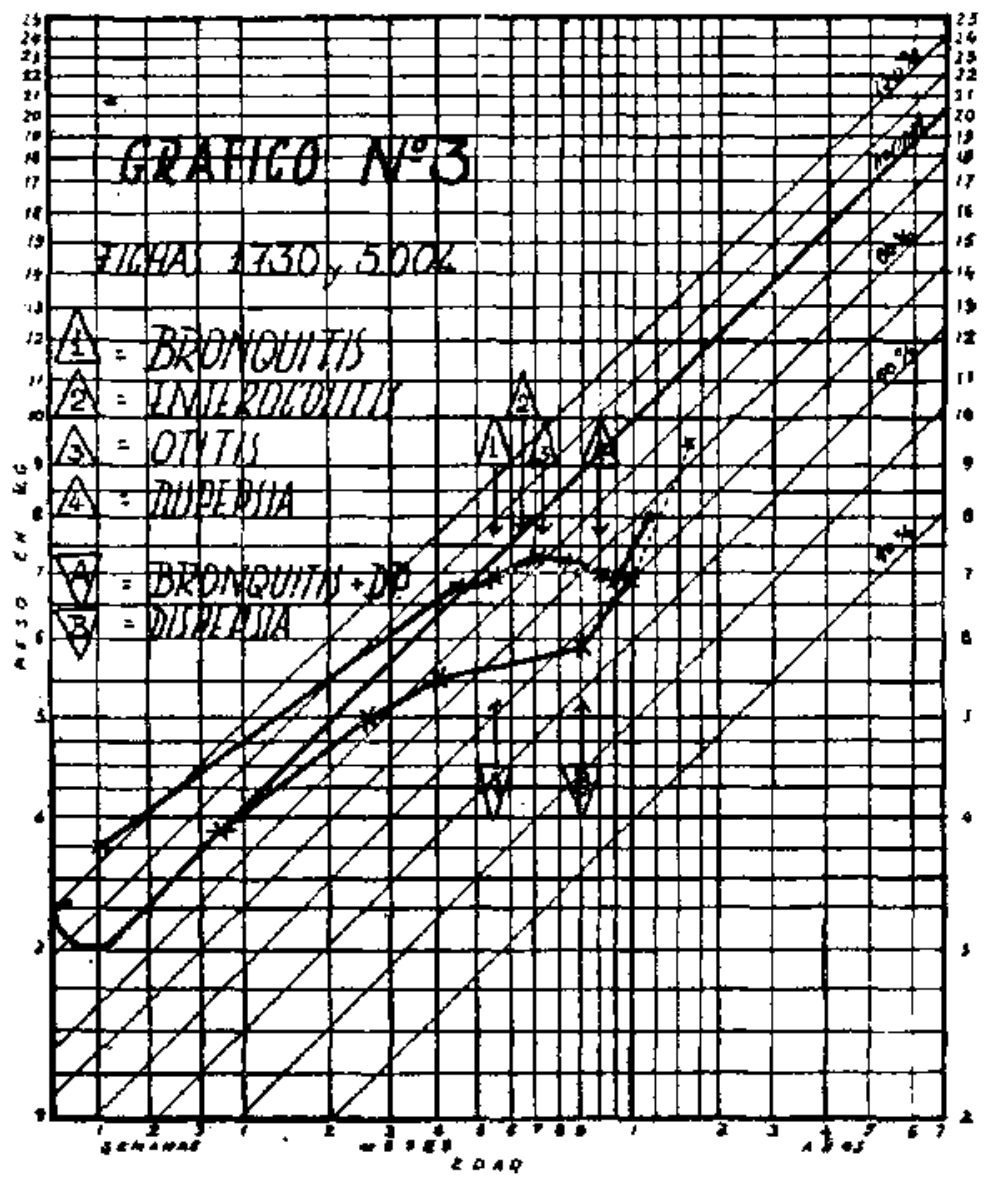

Hubo 5 madres en esta situación a la que pudo agregarse sólo un caso de abandono total del padre a su familia. Por supuesto que en varios casos el factor económico se presentó intimamente ligado a problemas de personalidad de la madre 0 de otro tipo, o fué la causa de estos problemas, especialmente desmoralización $\mathrm{y}$ estados de angustia en los familiares.

La acción efectuada en estos casos fué de resultados variables, más efectiva cuando se trataba de problema económico exclusivo y más difícil cuando a él se unían problemas de personalidad. En todas se otorgó gratuidad absoluta para toda clase de prestaciones del Servicio, En varios casos de cesantía, se pudo conseguir algún tipo de trabajo para el jefa del hogar $y$ otras veces trabajos de tipc casero para las madres. En el caso del inspector de Liceo se obtuvo la rápida terminación al trámite de pago de sus sueldos. Cuando se trataba de madres sol- teras la acción se dirigió a obtener la ayuda del padre o la aceptación de la madre en la casa de su familia.

2. Los problemas de personalidad de la madre $u$ otros familiares estuvieron presente en 23 de los 27 casos analizados. El problema más corriente fué la falta de conocimientos de las madres sobre la técnica de alimentación (17 casos). La acción fundamental en estas situaciones fué la educación intensiva, consiguiéndose buenos resultados en la mayoría. En 8 madres había negligencia y falta de interés evidentes, situación ligada casi siempre a mala situación económica, y más frecuente en el grupo de madres solteras. Estas madres reaccionaron en la medida que se solucionaron sus problemas económico-sociales.

Nueve madres presentaron problemas emocionales, consistentes la mayor parte de las veces en desmoralización o estados de angustia secundarios a la mala situa- 


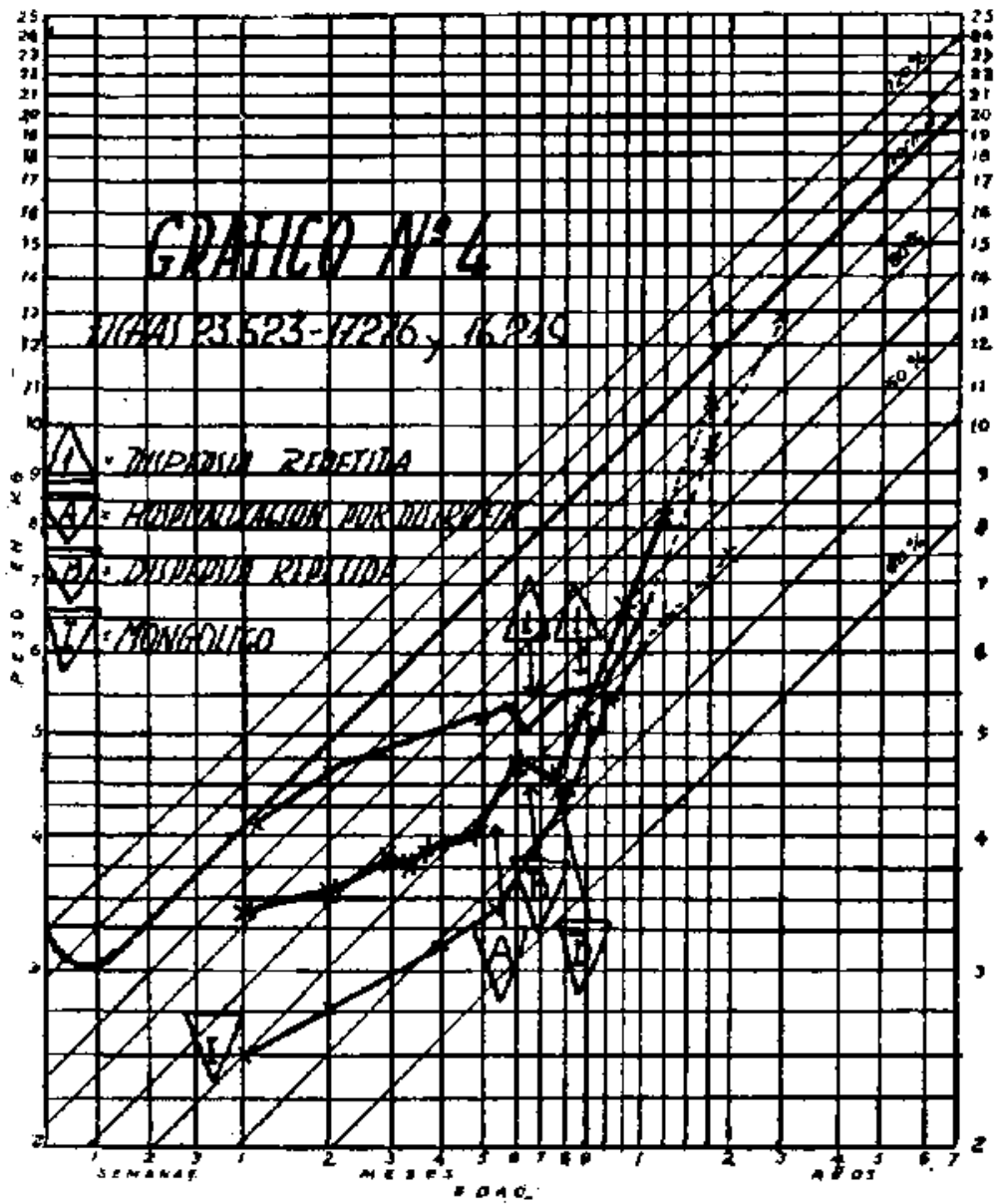

ción económica y que se corrigieron al mejorar ésta. Finalmente, en dos hubo sospechas bien fundadas de oligofrenia. Como caso extremo, tuvimos una madre soltera, en que se comprobó que persistentemente cometía errores intencionados en la alimentación del niño, al que finalmente asesinó arrojándolo a un canal.

De la revisión anterior, se deduce que las diferentes situaciones presentadas se encontraron simultáneamente en varias familias, y en que la acción de la enfermera debió combinar los intentos de soiución del problema económico, la educación y la psicoterapia.

3. Los factores distrofiantes derivados de situaciones previsionales o asistenciales fueron poco frecuentes, ya que la atención infantil se dió en forma gratuita y completa a prácticamente todos los niños, sin distinción de situación previsional. Hubo sólo 2 casos comprobados en que la familia no retiraba la leche entregada por el S. N. S., en un caso por simple negligencia y en el otro por preferir la madre cobrar el subsidio y comprar leche condensada, en cantidades insuficientes.

En todo caso, una de las primeras medidas en la mayoría de los lactantes distróficos analizados fué conceder gratuidad absoluta y hacer extensiva la entrega de leche a los hermanos mayores.

4. En igual forma, los factores ambientales no revistieron tampoco gran importancia, ya que la mayoría de nuestros niños habitaba en la población Simón Bolívar de la Corvi, en edificios de buena construcción, con agua potable y alcan tarillado, aunque eso sí, con aglomeración importante. Sólo en 4 casos las familias habitaban en viviendas insalubres.

5. Por último, en 2 casos no se encontró causa alguna que explicara la dis- 


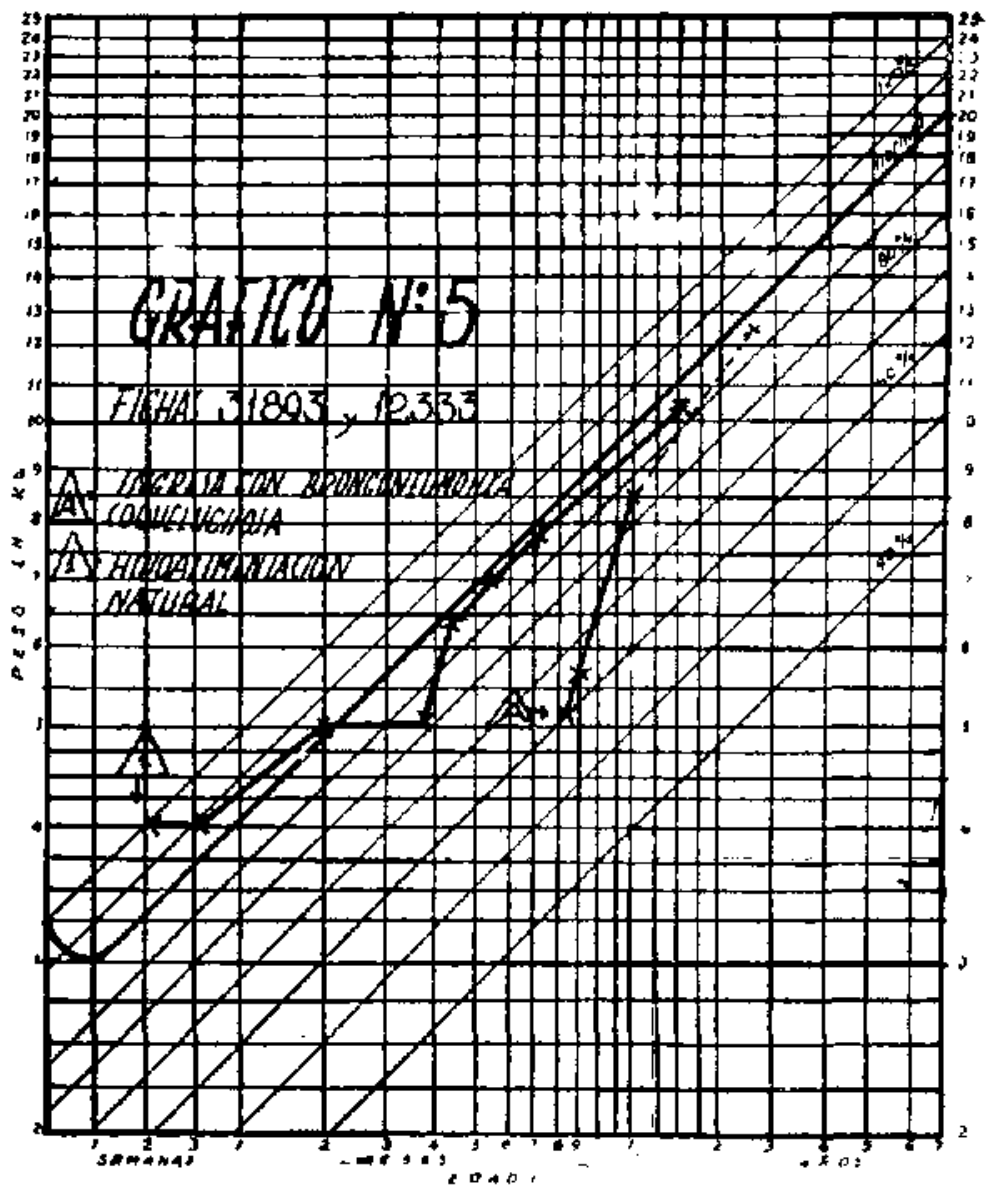

trofia, ya que se trataba de familias de posición económica relativamente buena y de madres responsables y con conocimientos satisfactorios. Es posible que la causa de la distrofia en ellos haya sido puramente médica.

En cuanto al resultado final obtenjdo en este grupo de niños distróficos, no hay información en 4 de ellos, y en los 23

TABLA NP 2

EVOLDCION Y PRONOSTICO DE I.A DISTROFIA FHN TN GRUPO DE 23 LACTANTLS INSCRITOS EN HI EX-CENTRO DE SALTD NQ $5-1957-1955$

\begin{tabular}{|c|c|c|}
\hline & $N 9$ & $\%$ \\
\hline 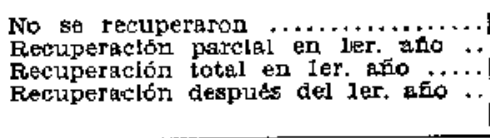 & $\begin{array}{l}7 \\
4 \\
4 \\
8\end{array}$ & $\begin{array}{l}30,0 \\
17,5 \\
27,5 \\
35,0\end{array}$ \\
\hline TOTAL $\ldots \ldots \ldots \ldots \ldots \ldots \ldots$ & 23 & 100 \\
\hline
\end{tabular}

restantes la evolución está expuesta en la Tabla N ? 2.

Comentario y conclusiones. Como criterio para conocer la frecuencia de la distrofia en nuestro grupo de lactantes, hemos usado la curva del peso, en relación con el gráfico confeccionado por el Dr. Mardones. Estamos conscientes que este criterio puede ser discutible, ya que no considera una serie de otros hechos que caracterizan la distrofia, como la disergia, los caracteres físicos, etc. Tiene sin embargo la ventaja que es un índice fácilmente apreciable y medible, aún para personal no médico. Es además, generalmente el signo más precoz, lo que permite dar el alerta ante un niño que disminuye su ritmo de crecimiento y actuar sobre él, antes que el proceso siga adelante.

Aceptando este criterio convencional, el uso de una curva de peso como la usada por nosotros, constituye un método gráfico extraordinariamente útil para orientar 
rápidamente sobre la evolución del proceso de crecimiento. La objeción hecha muchas veces que la confección de esta curva recarga excesivamente el trabajo médico no tiene validez, ya que ella puede quedar sin inconvenientes a carţo de una auxiliar de enfermería.

Insistiendo en lo dicho anteriormente, el porcentaje de $40 \%$ de lactantes que presentan alteraciones de su curva ponderal no corresponde al de distróficos. Muchos fueron descensos transitorios por cuadros mórbidos diversos, con rápida recuperación posterior. Otros fueron descensos demasiado discretos para considerarlos como índice de distrofia, siendo tal vez más correcto considerar el $23 \%$ de lactantes cuyos pesos descendieron bajo el $80 \%$ de lo normal como mejor representante de Ia cifra de distróficos. Es de advertir que los resultados obtenidos en el sector estudiado deben ser muy semejantes a lo que sucede en el resto de la Comuna Quinta Normal, ya que una comparación efectuada entre ellos de los promedios mensuales de aumento ponderal, no mostró diferencias.

El hecho más interesante de este trabajo es que el porcentaje de lactantes que habian sido catalogados como distróficos, según el criterio comentado, y que recuperaron parcial o totalmente su curva de progreso ponderal, alcanzó a $16 \%$ del total, o un $41 \%$ de los distróficos. Esta proporción de recuperados fué aún más alta en los distróficos avanzados, siendo de $54 \%$ y $64 \%$ en los grupos de niños con pesos bajo percentil 80 y 70 , respectivamente. Podríamos tal vez relacionar este hecho algo paradojal, con el hecho que los distróficios más avanzados recibieron una atención más intensiva de parte del Servicio. Esta experiencia es un buen estímulo para el trabaio en los Centros Infantiles ya que demuestra que es posib] corregir los trastornos del crecimiento, aunque estos sean avanzados, con tratamiento ambulatorio, en una buena proporción de lactantes.

En la $2^{\text {a }}$ parte de nuestro trabajo se presentaron en forma analítica Ios diver. sos factores económico-sociales que fú posible individualizar en los hogares de los niños distróficos. Es obvio que en la mayor parte de los grupos familiares thabía una combinación de ellos, pero varia veces era uno el que determinaba a lor otros. No constituye novedad que los 2 factores aislados más frecuentes son la falta de recursos económicos y la falta de conocimientos de las madres. De mayor interés resultó la comprobación de que hay un número importante de estas situaciones que pueden ser solucionadas, con los recursos con que cuenta corrientemente un Centro Infantil. E1 éxito en muchos casos, en que hay una evidente ignorancia de las familias, requiere sólo de una organización tal del trabajo de la enfermera, que puede concentrar su actividad sólo en los grupos de lactantes distróficos. En esta forma le es posible hacer una educación intensiva y controlada, que no es factible al repartir sus esfuerzos en gran número de familias. La falta de recursos económicos pudo también abordarse con cierto éxito, con mefidas sencillas de asistencia social. Una buena educación sanitaria y la solución parcial de otros problemas de personalidad de las madres.

Nuestra impresión final es, por todas estas razones, que si bien la distrofia es la resultante de las deficientes condiciones económico-sociales de una colectividad, y que el problema se resolverá en definitiva sólo cuando el nivel de vida de la población alcance un nivel satisfactorio, hay base para ser optimista sobre los resultados que un Centro Infantil puede obtener.

Para ello es necesario una buena planificación y administración del trabajo, $\mathrm{Y}$ un mínimo de linterés en estas labores. Si los recursos disponibles pueden además ser mayores que los nuestros, el resultado será aún mejor.

Finalmente, deseamos hacer resaltar dos conceptos:

a) Para obtener éxito en el maneio ambulatorio de las distrofias, el diagnóstico y la terapéutica deben abordarse tanto desde el punto de vista médico como económico-social. Por esto, es indispensable la estrecha colaboración del pediatra con la enfermera o asistente social correspondiente.

b) Dado el gran número de niños a11。 atienden Ios Centros Infantiles, es indispensable planificar el trabajo del personal en forma racional, concentrando esw fuerzos en los grupos más expuestos ? riesgo de la población, que son fundamentalmente los distróficos. 


\section{RESUMEN}

1. Se analizan las curvas ponderales de 158 lactantes menores de 1 año, controlados en un Centro Infantil, Ios que se clasifican en 4 tipos.

2. El porcentaje die lactantes que presentó descensos importantes de peso en el primer año de vida alicanzó a $\mathbf{4 0 \%}$, de los cuales a su vez el $41 \%$ presentó luego una recuperación parcial o total.

3. En un grupo de 27 lactantes distró ficos se efectuó un análisis de los factores económico-sociales presentes, y se demostraron como los más importantes la falta de recursos económicos y los problemas de personalidad de las madres. En el primer aspecto lo más corriente fué la cosantía del jefe del hogar o la madre soltera abandonada. En el 2 , la ignorancia sobre cuidados del niño.

4. La acción de la enfermera sanitaria fué eficaz en solucionar estos problemas en un número importante de casos. Consistió fundamentalmente en educación sanitaria intensiva, ayudar en la solución de algunos problemas económicos y orientar y estimular a las madres.

5. Se comentan los resultados obtenidos y se proponen algunas conclusiones.

\section{SUMMARY}

STUDY OF INDICENDE AND SOCIAL-ECONOMIC CAUSES OF DISTROPHY AT A CENTER OF INFANT CARE

Ponderal curves of 158 infanis, under a year, controlled at an Infant care Center, are analized being clasified in 4 types.

The percentage of infants who presented important loss in weight, in their first year, reached a $40 \%$. Of this $40 \%, 41 \%$ soon presented total or partial recovery.

In a group of 27 disirophic infants, an analisys of the social-economic factors was made, being the most important the lack of economical resources, and the mother's personality problems. The first one due to unemployment of the head of the family, or the abandonned unmaried mother. The second to ignorance of infant (i)".

In a important number of cases the ac tion of the sanitary nurse was effective. It was based fundamentally on intenssive sanitary education, in helping to find some solution to the economic problem, and in guiding and stimulating the mother.

The results obtained are commented and some conclusions are proposed.

\section{ZUSAMMENFASSUNG}

ETNE STUDIE ÏBFR DIE HAUFIGKEIT UND DIE WIRTSCHAFTLICH - SOZIALEN URSACHEN DER DYSTROPHIE IN EINER KINDERBERATUNGSSTELLE

Die Gewichtskurven von 158 säuglingen von unter $1 \mathrm{Jahr}$, die in einer Kinderberatunsstelle kontrolliert wurden, werden analysiert und in 4 Typen klassifiziert.

Der Prozentsatz der Säuglingen, die im ersten Lebensjahr erhebliche Gewichtsab. nahmen zeigten, betrug $40 \% ; 41 \%$ von ihnen erholten sich im weiteren Verlauf teilweise oder völlig.

Bei einer Gruppe von 27 dystrophischen Säuglingen wurde eine Analyse der bestehenden wirtschaftlich-sozialen Faktoren vorgenommen, und es erwiesen sich als die wichtigsten Faktoren der Mangel an Geldmitteln und die Persönlichkeits-Probleme der Mütter. In der ersten Hinsicht war en die Arbeitslosihkeit des Haushaltungsvorstands oder der im Stiche gelassenen ledigen Mutter das üblichste, in der zweiten Hinsicht, die Unwissenheit auf dem Gebiete der Kinderpflege.

In einer erheblichen Zahl der Fälle war die Mitarbeit der Fürsorgeschwester bei der Lösung dieser Probleme von Erfolg. Sie bestand im wesentlichen in intensiver Erziehung zu hygienischem Verhalten, in der Hilfe bei Lösung einiger wirtschaftlicher Probleme und in Belehrung und Ansporn für die Mütter.

Die erzielten Resultate werden kommentiert und einige daraus zu ziehende Folgerungen in Vorschlag gebracht.

\section{BIBLIOGRAFÍA}

1.-SCROGG su aspecto médico-saclal. Rev. Ch. de Pedlatrla. $19: 1,1948$.

2.-MENEGHILLL, J. y col. - Estudlos en el lactante distroflco, Rev, Ch. de Pedlatría. 19:61, 1948.

3-ARIZTIA A. Z ZENTKNNO, T, - Trastornos nutr1tivos crónicos en el lactante. Rev. Ch. de Pediatría. $19: 171$, 1948 .

4.-BEHM, H. y col. - Problemas de namenclatura en Ia clasificacion de las defunctomes por trastorros nutritivos del lactante. Rev. Ch. de Pediatria. $24: 273.1958$.

5.-DUARTE, A. - Causas soclales de la distrofta. Rev. Ch. cle Pediatria. $22: \% 9$, 1951.

6.-GAILO, A. y BELLO, A. - Engajo de una nueva técnlca de atención en un Centro Infantil. Rev. Ch. de Pedlatria. 30:335, 1959. 